\title{
¿Es solo una cuestión de austeridad? Crisis económica y políticas de género en España
}

\author{
Natalia PALEO \\ Investigadora predoctoral \\ Departamento de Ciencia Política y de la Administración \\ Universidade de Santiago de Compostela \\ nataliapaleo@yahoo.es \\ Alba ALONSO \\ Investigadora postdoctoral \\ Departamento de Ciencia Política y de la Administración \\ Universidade de Santiago de Compostela \\ alba.alonso.alvarez@gmail.com
}

Recibido: 12.06 .2014

Aceptado: 22.01.2015

\section{RESUMEN:}

En España las políticas de género han sufrido un considerable retroceso en los últimos años. La crisis económica y la necesidad de desarrollar políticas de austeridad han sido presentadas como las razones principales para realizar importantes recortes en sus presupuestos y para eliminar diversos organismos. Paralelamente, la agenda de igualdad también ha sido reorientada, pasando a ocupar una posición central la promoción de la maternidad. Aunque estas nuevas prioridades políticas emergen en un contexto de crisis, acontecen en paralelo con la llegada al poder de los partidos conservadores en el gobierno estatal y en múltiples gobiernos regionales. En este sentido invitan a revisar la literatura sobre políticas de género y el color de los partidos políticos en el gobierno y a explorar la influencia de esta variable. A través del análisis de todas las Comunidades Autónomas y del gobierno estatal, este artículo mostrará el impacto de la presencia de los partidos conservadores en el poder, no solo en términos de pérdida de recursos sino también, y más importante, en términos del desarrollo de una agenda política focalizada en las mujeres como madres y cuidadoras. Para ello, en primer lugar, a través de un análisis de regresión logística, se cuantificará la influencia de dicha variable en los presupuestos en materia de igualdad de género de ambos niveles de gobierno para el periodo 20022014. En segundo lugar, a través de un análisis cualitativo se revisará su influencia en la evolución de la agenda política y de la estructura institucional durante ese mismo lapso de tiempo. De este modo, se mostrará que a pesar de que los retrocesos tienen lugar esencialmente en periodo de crisis, covarían de manera clara con la presencia de gobiernos conservadores. Todo ello permite concluir que la crisis económica actúa como una ventana de oportunidad para poner en cuestión un área competencial que había experimentado una creciente expansión en los últimos 20 años.

Palabras clave: Políticas de Igualdad; España: Crisis económica; Partidos políticos 


\title{
¿Is It Just a Matter of Austerity? The Economic Crisis and Gender Equality Policies in Spain
}

\begin{abstract}
:
Gender equality policies in Spain have experienced considerable setbacks in the last few years. The economic crisis and the need to implement austerity policies appear as the main reasons behind the huge budget-cuts and the elimination of some equality machineries. Additionally, the equality agenda has been reoriented, with maternity related issues occupying a central position. Even though these new policy priorities emerge during the crisis, they may well be related to the fact that conservative parties have taken office both at the state and the regional level. Thus, recent events invite revisiting literature on gender policies and the color of parties in government in order to explore the influence of such variable. Thanks to the analysis of all the Autonomous Communities and the central government, this article shows the impact of the presence of conservative cabinets not only in terms of a decrease of the resources dedicated to gender policies, but also in terms of the promotion of a new political agenda based on the roles of women as mothers and carers. For so doing, first, a logistic regression analysis helps capture the incidence of this variable with regard to the budgets spent by both levels of government during the period 2002-2014. Second, a qualitative analysis assesses its impact on the emergence of a new policy agenda and the shifts that have occurred in relation to the equality architecture during this same lapse of time. Consequently, this article reveals that even though most setbacks have taken place during the economic crisis, they clearly covariate with the presence of conservative parties in office. This fact allows concluding that the crisis has acted as a window of opportunity which has permitted to call into question a policy sector that had experienced a 20 -year expansion period.
\end{abstract}

Key words: Equality Policies; Spain: Economic Crisis; Political Parties.

\section{INTRODUCCIÓN}

Las políticas de género en España han experimentado una progresiva expansión y consolidación a lo largo de las últimas décadas, de manera que están presentes en muy diversos niveles de gobierno. De este modo, dichas políticas han dado lugar a un área competencial diferenciada que presenta organismos, estrategias o instrumentos propios, además de una agenda política específica. A pesar de su carácter sectorial, estas políticas han contado con una marcada y creciente vocación transversal por la cual el mainstreaming ha devenido en estrategia de actuación clave (Alonso, 2015). Igualmente, los organismos de igualdad han ampliado progresivamente sus ámbitos de actuación, fortaleciendo su papel de agenda-setters e incidiendo en la necesidad de implicar a todas las áreas de gobierno en la lucha por la igualdad. Con ello, han tenido impacto en muy diversas áreas que resultan centrales para la vida de las mujeres.

En contraste con todos estos avances, durante los años más recientes se han comenzado a poner en cuestión algunos de los aspectos claves de las políticas de género y se han producido notables retrocesos. Amparándose en la necesidad de 
aplicar políticas de austeridad, se han llevado a cabo importantes recortes en los recursos dedicados a estas políticas, mientras que razones de eficacia y eficiencia han justificado la supresión de organismos de igualdad. Paralelamente, se han acometido cambios relevantes en la dimensión legislativa. Así, por un lado, se ha paralizado la aprobación de diversas leyes de igualdad autonómicas y, por otro, se ha introducido en la agenda política la protección de la mujer embarazada y del derecho a la vida. Este tipo de temáticas se han incorporado en normas que suponen un cambio de paradigma en relación con las leyes precedentes, dado que se focalizan en el rol de las mujeres como madres, limitan su capacidad de decisión respecto a la interrupción del embarazo y obvian completamente la transformación de los roles de género. De manera complementaria, el gobierno central inició un proceso de reforma de la ley del aborto con el objeto de restringir notablemente este derecho.

Si bien todos estos retrocesos han tenido lugar durante un periodo de crisis económica, exceden los recortes meramente presupuestarios. Como consecuencia, resulta difícil enmarcarlos como simples efectos de la implementación de políticas de austeridad. Por el contrario, se observa que buena parte de estas modificaciones tienen lugar tras la llegada al poder de partidos conservadores tanto a nivel estatal como autonómico, lo cual podría apuntar a la relación entre el partido político gobernante y los retrocesos llevados a cabo. En este sentido, a través de este trabajo se quiere profundizar en la capacidad explicativa de ambas variables -crisis económica e ideología del partido político en el gobierno- con el propósito de evidenciar su impacto en el desarrollo de las políticas de género. Para ello se abordarán preguntas como las siguientes: ¿Constituye la crisis económica el factor que explica la presencia de retrocesos en materia de actuaciones para promover la igualdad? ¿Son éstos solamente una cuestión de austeridad? ¿Tiene, por el contrario, influencia el color del partido político que se encuentra en el gobierno? En ese caso, ¿puede representar la crisis económica una ventana de oportunidad gracias a la cual se frene o ponga en cuestión la expansión de esta área competencial?

Con el objeto de responder a este tipo de preguntas, este trabajo explora de modo pionero y tentativo los retrocesos en materia de políticas de igualdad en España -variable dependiente-, así como su vinculación con dos cambios recientes y cruciales: la llegada de gobiernos conservadores al poder y la irrupción de un contexto de crisis -variables independientes-. Con esa finalidad, se aborda el análisis de las actuaciones en materia de igualdad durante el periodo 2002-2014, el cual incluye épocas tanto de bonanza como de profundas restricciones presupuestarias. El artículo comienza con una breve revisión de la literatura específica en torno al desarrollo de las políticas de género y sus factores explicativos. Seguidamente, se describe la metodología empleada, que combina las 
técnicas de tipo cuantitativo y cualitativo y que comprende el análisis de las políticas de género en el nivel estatal y autonómico. Para concluir, se abordan tres dimensiones centrales de las políticas de género -los presupuestos, la legislación en materia de igualdad y los organismos de igualdad-. Para cada una de ellas se expone la evolución temporal, y se explora la incidencia de los factores explicativos seleccionados. Con todo ello, el texto contribuye a poner de manifiesto las importantes involuciones experimentadas en este ámbito de actuación, a la vez que se evidencia la pertinencia de explorar el impacto de dos variables que habían perdido cierto protagonismo en los debates de la disciplina.

\section{FACTORES EXPLICATIVOS DE LAS POLÍTICAS DE GÉNERO}

En paralelo al innegable surgimiento y consolidación de las políticas de género en múltiples países occidentales, ha emergido un fructífero campo de investigación que ha permitido explorar cómo, cuándo y por qué se han desarrollado este tipo de políticas públicas. Este tiene como resultado un amplio conjunto de estudios que han puesto de manifiesto al menos dos aspectos clave. En primer lugar, han evidenciado la evolución temporal de las políticas de igualdad, apuntando a la aprobación de legislación específica y de planes de actuación, y a la creación de instituciones con la misión de mejorar la situación de las mujeres como sus principales resultados. De hecho, estas medidas constituyen, junto con el establecimiento de presupuestos específicos, tres de los indicadores clave del surgimiento de esta área competencial y, como tales, son consideradas como variables dependientes en múltiples trabajos.

En segundo lugar, y de forma paralela, la literatura ha mostrado gran interés por identificar las variables que explican su rápido desarrollo en las últimas décadas. Por una parte, destacan aquellos estudios que centran la atención en el papel de determinados actores (agency). Muchos de ellos enfatizan el rol clave de agentes centrales del subsistema de las políticas de género, como son los organismos de igualdad o el movimiento de mujeres, que se han mostrado eficaces en la consecución de cambios en diferentes ámbitos de actuación (McBride y Mazur, 2010). El concepto de masa crítica, y más recientemente el de actores críticos, ha generado conclusiones análogas, emergiendo como factores explicativos de muy diversos avances en materia de igualdad (Childs y Krook, 2009). Asimismo, la presencia de partidos políticos de izquierda en el gobierno ha constituido también una variable de enorme importancia. Se ha podido evidenciar que constituye una condición favorable para el desarrollo de medidas relativas a la igualdad de género (McBride y Mazur, 2010). De hecho, en diversos casos de estudio se ha entendido que la llegada de la izquierda al poder representó una estructura de oportunidad para el movimiento feminista y de mujeres, de manera que facilitó que este tuviera influencia en el proceso político (Chappel, 2000). A su vez, se han identificado 
ejemplos en los cuales la presencia de retrocesos se ha producido como resultado de la entrada en el gobierno de partidos conservadores (Hankivski, 2008). No obstante, la variable color del partido político continúa siendo cuestionada y se matiza que no representa en ningún caso una condición suficiente para la presencia de avances (Mazur, 2003).

En paralelo al estudio del papel de diversos tipos de actores, se ha apostado también por explorar la incidencia de factores relativos a la estructura (structure). La pertinencia de incorporar esta clase de elementos se debe a la creciente importancia de fenómenos como los cambios en la distribución territorial del poder derivados de los crecientes procesos de federalización o de integración supranacional experimentados en ciertos países (Haussman et al, 2010). Estos han incrementado la pertinencia de explorar el impacto, por ejemplo, de los procesos de aprendizaje entre territorios y niveles de gobierno, y han dado lugar a destacables líneas de investigación relativas a la europeización o a la incidencia de los modelos federales (Lombardo y Forest, 2012; Krook y Mackay, 2011).

Igualmente, en las últimas décadas, también se ha prestado cierta atención al papel que juegan las crisis económicas en la promoción de la igualdad entre mujeres y hombres. Así, a partir de la década de los 70 emergió una abundante literatura sobre el impacto de género de las crisis económicas que estaban teniendo lugar en múltiples países recién industrializados y en desarrollo. En este sentido, las economistas feministas pusieron el foco de atención en los costes sociales específicos para las mujeres y para la igualdad de género de los programas de estabilización económica y de ajuste estructural que habían sido promovidos por el Fondo Monetario Internacional (FMI) en dichos países (Karamessini, 2013). De forma general, los análisis realizados muestran que durante las etapas de crisis económica se producen con frecuencia notables retrocesos en los avances en materia de igualdad conseguidos en épocas de bonanza (Gálvez y Rodríguez, 2013; Gálvez, 2012). Se ha indicado que para impedir la presencia de estos retrocesos resulta fundamental incluir la perspectiva de género en las políticas públicas específicas que se diseñen para hacer frente a las dificultades económicofinancieras. Por lo que se refiere a la crisis actual, los estudios existentes coinciden en señalar que las políticas de austeridad que se han llevado a cabo en la UE no han tenido en cuenta la perspectiva de género (European's Women's Lobby, 2009; Harcourt, 2009) y que la estrategia del mainstreaming no ha sido por lo tanto aplicada (Karamessini y Rubery, 2013). Asimismo, existen ya valoraciones preliminares sobre el impacto negativo que han tenido dichas políticas sobre la situación de las mujeres (Bettio et al., 2012; European Women's Lobby, 2012; King y Sweetman, 2010) y también sobre el desarrollo de las políticas referidas a la promoción de la igualdad. Estas han experimentado una notable involución, habiendo sido desplazadas de la agenda política en algunas ocasiones (Karamessini 
y Rubery, 2013). Además, si bien algunos de los recortes sufridos por esta área competencial - como por ejemplo la eliminación de los organismos de igualdadson presentados como medidas de reducción del gasto, en realidad el ahorro que suponen es tan insignificante que las motivaciones ideológicas han de ser también tenidas en cuenta (Ibídem). De hecho, existen estudios pioneros que ponen el foco de atención en el papel de los partidos conservadores como principales promotores de dichos recortes (Lang y Sauer, 2012).

Aquellos análisis que se han ocupado del caso español no han permanecido ajenos a la importancia de todos estos factores y han apostado por explorar también su incidencia en el desarrollo de las políticas de género. En este sentido, existen análisis referidos a la influencia de los organismos de igualdad (Valiente, 2007; Bustelo, 2004), de la presencia política de las mujeres (Threlfall, 2007; Paleo, 2013) o del modelo territorial (Bustelo, 2014; Alonso, 2015). El contexto actual invita, no obstante, a centrar la atención en dos elementos que o bien no habían sido analizados con anterioridad, o bien habían perdido relevancia. En primer término, la crisis económica actual ha coincidido en el tiempo con el momento de mayor expansión de las políticas de género en España, cuando estas habían sido generalizadas a todos los niveles de gobierno. Las valoraciones existentes hasta el momento han señalado que, al igual que sucede en otros niveles de gobierno, las políticas de austeridad implementadas para hacerle frente no han tenido en cuenta la perspectiva de género (Gálvez y Rodríguez, 2013). Así, desde el año 2010 se han estado llevado a cabo toda una serie de reformas - eliminación de organismos de igualdad, retraso de la extensión del permiso de paternidad, reforma del sistema de pensiones, suspensión del desarrollo de la ley de dependencia, etc.- que tienen un claro impacto negativo en la igualdad de género (González y Segales, 2013; De Villora, 2011). Sin embargo, resulta todavía necesario analizar en profundidad los retrocesos que han tenido lugar respecto a las políticas de género como área sectorial clave y valorar adecuadamente la influencia que ha tenido la crisis económica en los mismos.

En segundo lugar, los años más recientes han sido testigo también de un cambio en lo relativo al color político de los gobiernos central y de diversas autonomías. La literatura referida al caso español ha señalado en repetidas ocasiones el importante papel jugado por los partidos de izquierda, y en especial por el Partido Socialista (PSOE), en cambios clave como la creación de organismos de igualdad (Ortbals, 2008; Ortbals y Bustelo, 2007; Valiente, 1994) o el incremento de la representación descriptiva de las mujeres (Threlfall, 2007). No obstante, estas conclusiones se han matizado con argumentos que señalan que no representa una condición suficiente para la presencia de avances (Linos, 2003) o que las políticas de género constituyen ya una política de Estado, de modo que no son puestas en cuestión por los partidos conservadores (Astelarra, 2006). La presencia de diversos 
ejemplos de cambio en el color político del gobierno en los últimos lustros permitirá explorar de nuevo la capacidad explicativa de dicha variable y contribuir a abordar una vez más la una provocativa pregunta: "are socialists a woman's best friend?" (Linos, 2003: 438).

\section{METODOLOGÍA}

El objetivo general que persigue este trabajo consiste por lo tanto en explorar en qué medida la crisis económica y el color de los partidos políticos en el gobierno han tenido impacto en algunos de los retrocesos que se han producido recientemente en las políticas de género en España. Concretamente, dos son las hipótesis de partida: a) en contextos de crisis económica es más probable que se produzcan retrocesos en las políticas de género que en contextos de bonanza; b) en contextos de crisis económica, cuando gobierna un partido conservador es más probable que se produzcan retrocesos en las políticas de género que cuando lo hace un partido político de izquierda o de centro-izquierda.

Las políticas de género a nivel estatal y en las 17 Comunidades Autónomas (CCAA) para el periodo 2002-2014 representan la variable dependiente del estudio. Estas han sido definidas como aquellas medidas llevadas a cabo por los gobiernos para promover la igualdad entre mujeres y hombres, mejorar la situación socioeconómica de las primeras y erradicar las jerarquías de género. Teniendo en cuenta tanto esta definición como la literatura revisada, estas políticas han sido operacionalizadas a través de tres dimensiones:

1) Presupuestos destinados a las políticas de género: se refiere a las cantidades económicas totales que destinaron anualmente los diferentes gobiernos a esta política sectorial durante el periodo 2002-2014. Se ha considerado que se producían retrocesos si disminuía la cantidad económica dedicada a estas políticas. La fuente de datos utilizada fueron los presupuestos publicados en el Boletín Oficial del Estado y los Boletines Oficiales Autonómicos ${ }^{1}$, habiéndose generado en total 192 casos, un $89 \%$ de total de casos posibles $(\mathrm{N}=216)^{2}$.

\footnotetext{
${ }^{1}$ En general, los presupuestos incluyen programas específicos dedicados a la promoción de la mujer o de la igualdad entre mujeres y hombres. Además, en ejemplos como el estatal existen programas presupuestarios dirigidos en exclusiva a la lucha contra la violencia de género.

${ }_{2}$ El total de casos posibles $(\mathrm{N}=216)$ se corresponde con el presupuesto del Estado y de las Comunidades Autónomas durante cada uno de los 13 años analizados. Entre los 24 casos que no se han podido analizar, se encuentran los presupuestos de la Comunidad Autónoma de la Rioja durante todo el periodo (13 casos), puesto que no fue posible a través de los
} 
2) Legislación estatal y autonómica en materia de igualdad: hace referencia a la legislación relacionada específicamente con la defensa de los derechos de las mujeres, la promoción de la igualdad y la eliminación de las jerarquías de género que fue aprobada a nivel estatal y autonómico entre 2002 y 2014. Para analizar esta dimensión, por un lado, se han identificado todas las leyes en esta materia elaboradas durante el periodo analizado (tanto las leyes aprobadas como las propuestas de ley que no prosperaron) y por otro lado se ha analizado el contenido de las mismas. En este caso, se han considerado como retrocesos la no aprobación de algunas leyes cuyo proceso de tramitación ya había comenzado y la aprobación de otras, que si bien se dirigen a las mujeres, no promueven la igualdad de género. La fuente de información utilizada han sido los Boletines Oficiales de ambos niveles de gobierno y los boletines parlamentarios.

3) Organismos de igualdad: hace referencia a los organismos de ámbito estatal y autonómico que tienen oficialmente la misión específica de llevar a cabo las políticas de género en un territorio determinado. En este caso, para analizar esta dimensión se han examinado los procesos de creación y eliminación de dichos organismos durante el periodo analizado. La fuente de datos para analizar esta dimensión han sido de nuevo los Boletines Oficiales, así como la literatura secundaria.

Estas tres dimensiones se focalizan en las políticas de igualdad como políticas sectoriales y por lo tanto no recogen la riqueza y variedad de actuaciones gubernamentales que pueden tener impacto en la vida de las mujeres (políticas sociales, educativas, etc.). Sin embargo, incluyen los elementos centrales de esta área competencial, los cuales resultan cruciales para promover la inclusión de la perspectiva de género en otros ámbitos de actuación de especial relevancia (Alonso, 2015).

Por otro lado, la crisis económica y el color de los partidos políticos gobernantes en ambos niveles de gobierno constituyen las variables independientes. La variable 'crisis económica', se refiere a la crisis en la economía mundial producida a partir del año 2008. En el caso de España, las principales consecuencias de esta comenzaron a notarse durante ese mismo año, siendo sus primeras

documentos públicos encontrar la información necesaria. Asimismo, no consta información de los presupuestos respecto a años concretos para la Comunidad Valenciana (3 casos), la Comunidad Foral de Navarra (4 casos) y la Región de Murcia (5 casos), debido a que determinados cambios en los programas presupuestarios en esos años concretos impedían su comparación con los presupuestos del resto del periodo analizado. 
manifestaciones el rápido incremento del desempleo, la ejecución de numerosos expedientes de regulación de empleo (ERE) o de suspensión de pagos. Para medir la influencia de esta variable se ha distinguido entre un periodo de bonanza económica (2002-2007) y uno de crisis (2008-2014) ${ }^{3}$.

En lo que respecta a la variable 'color del partido político', esta hace referencia a la ubicación ideológica en el eje derecha-izquierda de los partidos gobernantes a nivel central y autonómico. Con el objeto de medir esta variable, se han empleado los resultados electorales disponibles en la página web del Ministerio de Interior para identificar los partidos políticos o coaliciones que ocuparon los gobiernos del Estado y de las Comunidades Autónomas durante el periodo analizado. En segundo lugar, a partir de diversos estudios postelectorales del Centro de Investigaciones Sociológicas (CIS), que incluyen un análisis de la ubicación ideológica de los partidos, se ha procedido a atribuir a cada partido una puntuación entre 0 y 10 , donde 0 correspondía al extremo de las posiciones de izquierda y 10 al extremo de las posiciones de derecha. En el caso de las coaliciones, tomando como referencia las puntuaciones de cada uno de los partidos que las conformaban, se ha realizado una media ponderada, distinguiendo entre primer, segundo y, si procedía, tercer partido, según los escaños que tuviesen cada uno de los partidos de la coalición. Una vez obtenidas las puntuaciones de cada partido y coalición, se llevó a cabo una categorización, de tal forma que se han diseñado cinco posiciones ideológicas posibles: Izquierda (puntuaciones 1 y 2), Centro-Izquierda (puntuaciones 3 y 4), Centro (5 y 6), Centro- Derecha (7 y 8), y Derecha (9 y 10). ${ }^{i}$

Finalmente, respecto a las técnicas de análisis utilizadas para examinar la relación entre estas variables, destaca el hecho de que se han combinado técnicas de tipo cuantitativo y cualitativo. Así, una primera parte del trabajo ha consistido en analizar cuantitativamente su influencia en los presupuestos dedicados a las políticas de género en el Estado y en las Comunidades Autónomas. Concretamente, las técnicas de análisis que se han utilizado fueron el análisis de frecuencias y el análisis de regresión logística. A través de la primera de ellas se realizó una aproximación general al comportamiento de la variable dependiente en función de las variables independientes y a través de la segunda se cuantificó exactamente la relación existente entre ellas.

En una segunda parte de este trabajo, y gracias a un análisis de tipo cualitativo, se exploró su influencia en la aprobación de legislación directamente

\footnotetext{
${ }^{3}$ A la hora de analizar la influencia de esta variable en los presupuestos, se considera que el periodo de crisis comienza en el 2009 dado que los presupuestos normalmente se elaboran el año anterior a su entrada en vigor.
} 
relacionada con los derechos de las mujeres y en la existencia de los denominados organismos de igualdad. Asimismo, se realizó una aproximación al contenido de la normativa, para lo cual se tuvieron en cuenta las sugerencias realizadas desde el análisis de marcos interpretativos aplicado al estudio de las políticas de género (Verloo, 2007). El propósito de este tipo de análisis ha sido desvelar el concepto de igualdad que subyace a las mismas, y determinar su potencialidad para conseguir una transformación de las relaciones de género. En esta parte del estudio, de nuevo se contempló tanto el nivel estatal como el autonómico durante el periodo 20022014, aunque se focalizó la atención en aquellos cambios acontecidos en el periodo de crisis (2008-2014), los cuales pueden ser identificados de modo claro como retrocesos.

\section{RESULTADOS}

\subsection{La reducción presupuestaria}

La literatura sobre políticas públicas ha considerado de manera general que los recursos económicos representan un elemento decisivo para la consecución de los objetivos de cualquier política pública (Subirats et al, 2008). Asimismo, en los análisis referidos específicamente a las políticas de género también se ha apuntado con frecuencia que los presupuestos destinados a las mismas constituyen un indicador importante sobre el nivel de compromiso de un gobierno con la igualdad, así como un factor central en su proceso de institucionalización (Astelarra, 2005). En este sentido, diversas investigaciones han concluido que los recursos constituyen un factor esencial para determinar la efectividad de los organismos de igualdad (Squires, 2007). Es por ello que en el análisis de los retrocesos en las políticas de género se consideró relevante examinar los recortes que se han producido en los presupuestos dedicados a dichas actuaciones públicas, así como su relación con las variables seleccionadas.

\section{El impacto de la crisis económica}

En primer lugar, para explorar la incidencia de la crisis económica en los recursos dedicados a las políticas de género, resulta interesante comparar la evolución de los presupuestos destinados a dichas políticas durante los dos periodos seleccionados. En el gráfico siguiente se puede observar de manera clara que, mientras durante la primera etapa en el $90 \%$ de los casos analizados $(\mathrm{N}=90)$ el presupuesto dedicado a las políticas de género aumentó o se mantuvo constante, en el periodo de crisis económica este porcentaje se redujo en un $27,2 \%$ de los casos $(\mathrm{N}=25)$, habiendo un $72,8 \%$ de casos $(\mathrm{N}=67)$ en los cuales el presupuesto disminuyó. 
Gráfico 1: Variaciones en los presupuestos dedicados a las políticas de género en el Estado y las Comunidades Autónomas según la presencia o no de crisis económica

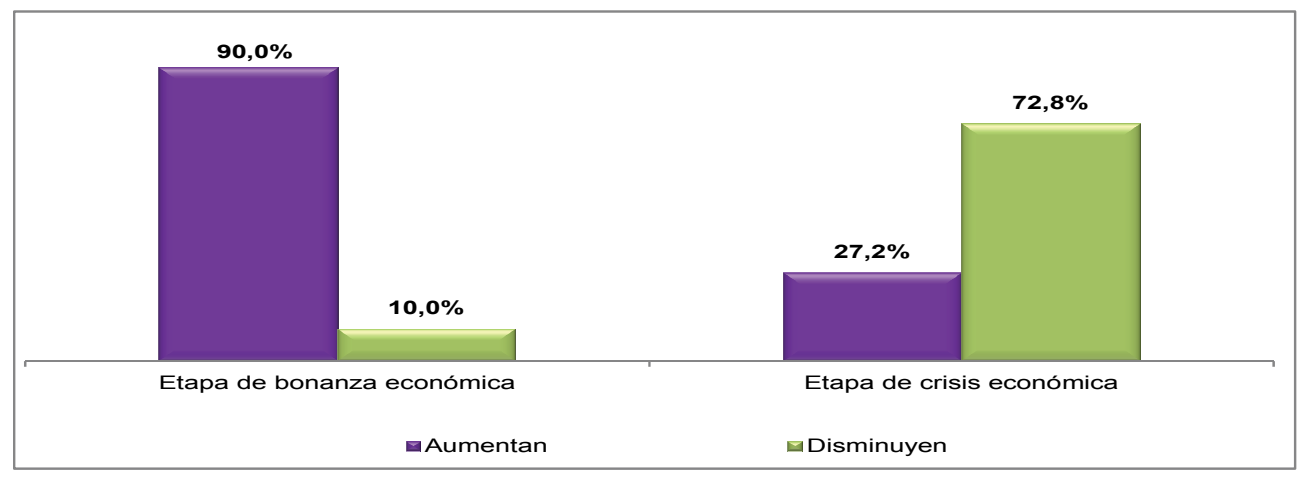

Este primer análisis de porcentajes parece indicar, por lo tanto, que la variable crisis económica influye de forma considerable en los retrocesos en materia de recursos. Asimismo, también se extrae esta misma conclusión al analizar las variaciones totales que se producen en los presupuestos ${ }^{4}$ del Estado y de las Comunidades Autónomas en cada uno de los periodos. Así, se observa que mientras que entre 2002 y 2008 en todos los casos estudiados el presupuesto dedicado a las políticas de género se incrementó considerablemente, en el periodo 2009-2014 el presupuesto disminuyó en todos los casos, excepto en la Comunidad Autónoma de Andalucía en la cual aumentó un 16,6\%.

4 Las variaciones en los presupuestos son aproximativas. En algunos casos (Navarra, Valencia, Murcia, Catalunya y Galicia) no ha sido posible conseguir la información relativa a los presupuestos de alguno de los años analizados o dicha información no era comparable. 
Gráfico 2: \% de variación en el presupuesto destinado a las políticas de género durante el periodo 2002-2008

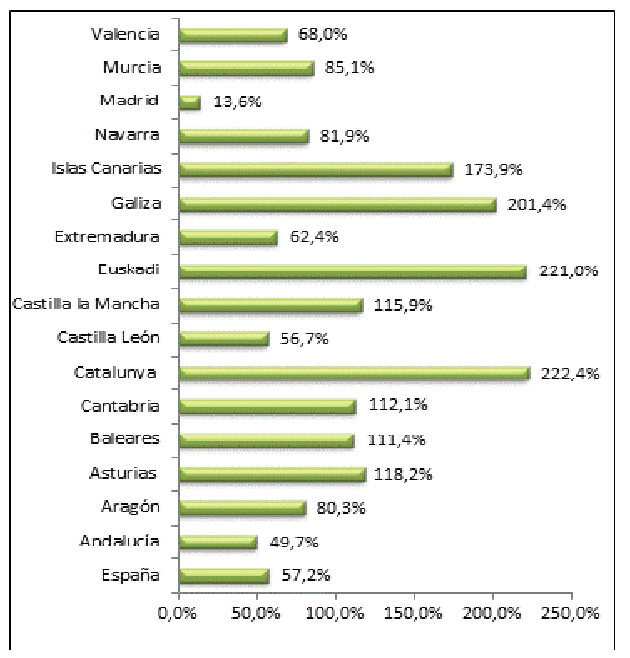

Gráfico 3: \% de variación en el presupuesto destinado a las políticas de género durante el periodo 2009-2014

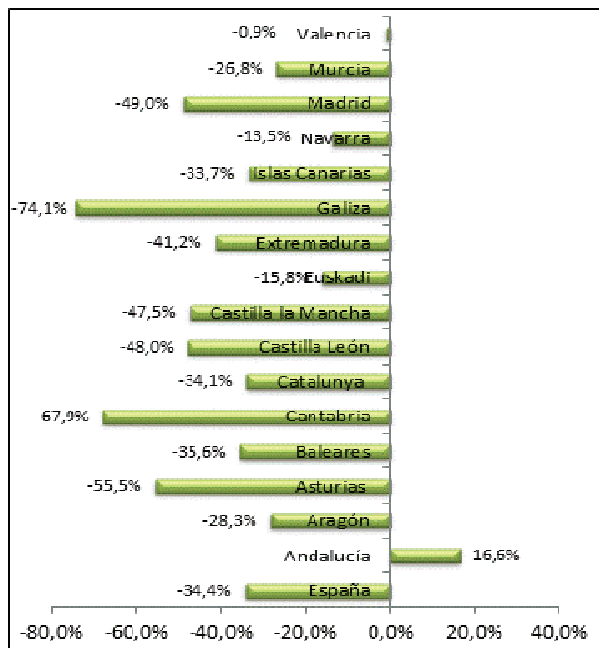

Para finalizar, un tercer paso para examinar la influencia de la crisis económica en los recursos dedicados a las políticas de género ha consistido en realizar un análisis de regresión logística binaria. De acuerdo con los resultados obtenidos, se puede concluir que esta variable tiene una relación estadísticamente significativa con el presupuesto dedicado a dichas políticas. Concretamente, los datos muestran que cuando está presente hay 24,12 veces más posibilidades de que el presupuesto dedicado a estas políticas se reduzca que en época de bonanza. Por otro lado, desde una perspectiva predictiva, los cálculos realizados muestran que la probabilidad de que se produzcan recortes en las políticas de género en contextos de crisis económicas equivale a un $99,5 \%$. 
Tabla 1: Resultados análisis de regresión logística

Presupuesto dedicado a las políticas de género

\begin{tabular}{|c|c|c|c|c|}
\hline & B & S.E. & Sig. & $\operatorname{Exp}(B)$ \\
\hline $\begin{array}{l}\text { Crisis económica } \\
\text { Ausencia de crisis económica ( }\end{array}$ & & & & \\
\hline Presencia de crisis económica & 3,18 & 0,41 & 0,00 & 24,12 \\
\hline Constante & $-2,20$ & 0,33 & 0,00 & 0,11 \\
\hline
\end{tabular}

\section{El impacto del partido político en el gobierno}

Para analizar la influencia del color del partido político, en primer lugar, se han observado las variaciones de los presupuestos en función de dicha variable durante los años de bonanza económica (2002-2008) y durante los años de crisis (2009-2014).

Tal y como se puede observar en el siguiente gráfico, durante el periodo de bonanza económica la mayoría de los partidos políticos con responsabilidades de gobierno incrementaron o mantuvieron constantes los presupuestos destinados a las políticas de género. El porcentaje más alto de aquellos partidos gobernantes que disminuyeron los presupuestos durante este periodo se ubican en una posición de centro-derecha, aunque las diferencias en relación a los partidos de centro-izquierda son mínimas. Además, se observa que entre los partidos ubicados ideológicamente en el centro no hay ninguno que disminuyese el presupuesto durante este periodo. 
Gráfico 4: Variaciones en el presupuesto en función de la ideología de los partidos políticos gobernantes durante el periodo de bonanza económica (2002-2008)

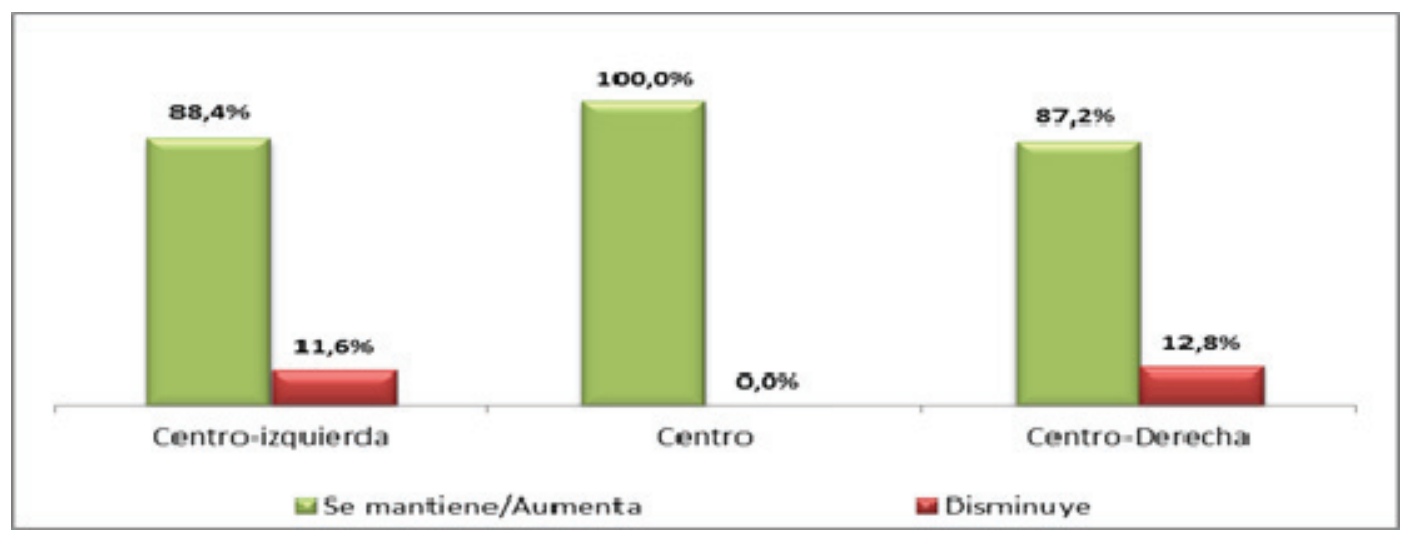

Por el contrario, durante el periodo de crisis económica la tendencia es inversa y se detectan diferencias notables en función de la posición ideológica de los partidos políticos gobernantes. Mientras entre todos los gobiernos con partidos de centro-derecha hay un $90,7 \%$ que disminuyeron el presupuesto destinado a las políticas de género, en el caso de los gobiernos con partidos de centro este porcentaje desciende hasta un $68,8 \%$ y entre los partidos de centro-izquierda a un $51,5 \%$. 
Gráfico 5: Variaciones en el presupuesto en función del color político del partido en el gobierno durante el periodo 2009-2014

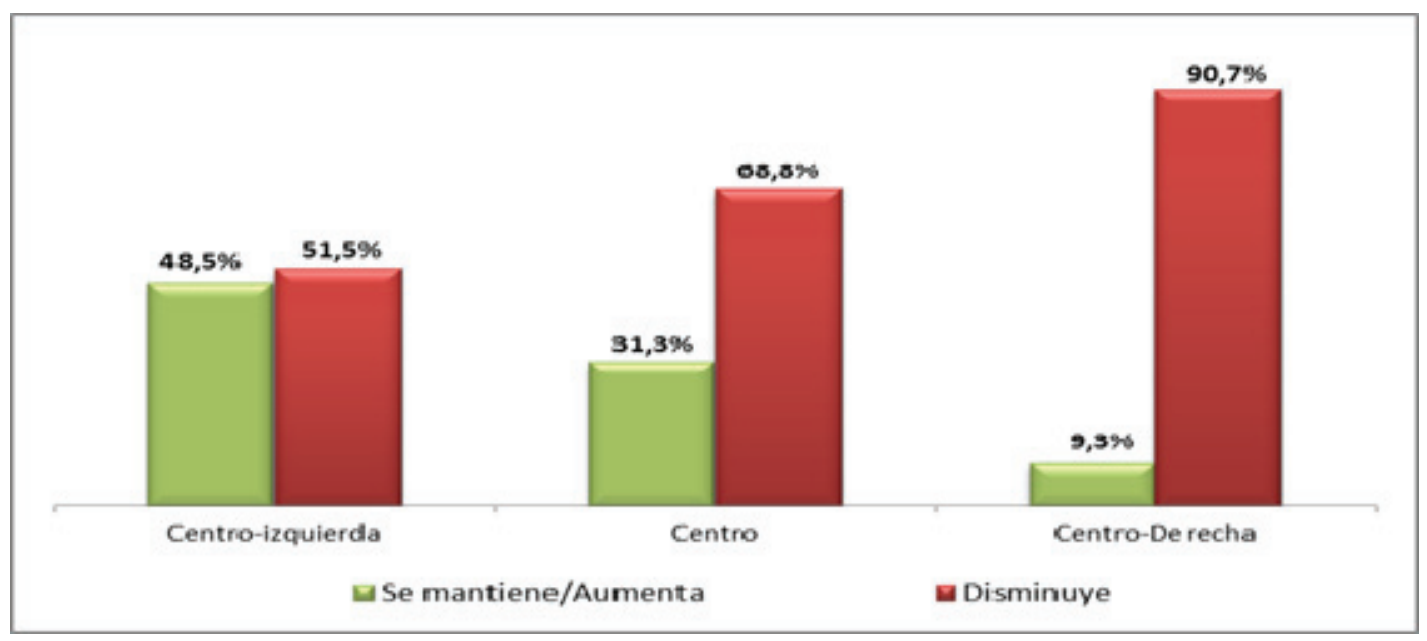

Estos datos ponen de manifiesto que si bien durante los periodos de bonanza económica la ideología del partido gobernante no influye o influye muy poco, en periodos de crisis económica esta variable adquiere relevancia, de tal forma que los recortes en los presupuestos de las políticas de género son realizados en mayor medida por los partidos de centro-derecha y de centro. De hecho, las diferencias entre los partidos o coaliciones de centro-izquierda y de centro-derecha son muy elevadas, aproximadamente de 39 puntos.

Por otro lado, los resultados del análisis de regresión muestran que, manteniendo constante los efectos de la crisis económica, la variable del color del partido político tiene una relación estadísticamente significativa con los recortes en el presupuesto. Así, tomando como referencia a los partidos o coaliciones de centroizquierda, se observa que cuando en los gobiernos hay un partido o una coalación de centro-derecha hay 4,15 veces más posibilidades de que se reduzcan los presupuestos dedicados a las políticas de género. Por otro lado, si calculamos la probabilidad de que disminuyan los presupuestos de las políticas de género cuando hay un gobierno centroderecha y se mantienen constantes los efectos de la crisis económica se observa que esta equivale a un $98,8 \%$. 
Tabla 2: Resultados análisis de regresión logística

Reducción del presupuesto dedicado a las políticas de género

\begin{tabular}{|c|c|c|c|c|}
\hline & B & S.E. & Sig. & $\operatorname{Exp}(B)$ \\
\hline \multicolumn{5}{|c|}{$\begin{array}{l}\text { Crisis económica } \\
\text { Ausencia de crisis económica (Ref.) }\end{array}$} \\
\hline $\begin{array}{l}\text { Presencia de crisis } \\
\text { económica }\end{array}$ & 3,37 & 0,45 & 0,00 & 29,22 \\
\hline \multicolumn{5}{|c|}{$\begin{array}{l}\text { Ideología del partido político en el gobierno } \\
\text { Partido de Centro Izquierda (Ref.) }\end{array}$} \\
\hline Partido Político & & & 0,00 & \\
\hline Partido de Centro & 0,10 & 0,56 & 0,86 & 1,11 \\
\hline Partido de Centro-Derecha & 1,42 & 0,46 & 0,00 & 4,15 \\
\hline Constante & $-2,96$ & 0,48 & 0,00 & 0,05 \\
\hline
\end{tabular}

Finalmente, resulta pertinente realizar un análisis únicamente para los casos del periodo de crisis, es decir, explorar la relación entre la ideología de los partidos políticos gobernantes y los recortes en los presupuestos de las políticas de género durante dicha etapa. Ello permite observar, en primer lugar, que la ideología del partido tiene una relación estadísticamente significativa con el presupuesto dedicado a las políticas de género. En segundo lugar, tomando como referencia a los partidos de centro izquierda, los datos indican que cuando hay un partido de centro-derecha en el gobierno hay 9,18 veces más posibilidades de que se recorten los presupuestos dedicados a las políticas de género. Además, también se observa que cuando hay un partido de centro en el gobierno las posibilidades de que se reduzcan los presupuestos aumentan 2,07 veces en relación a cuando hay un partido de centro-izquierda, aunque en este caso la relación no es estadísticamente significativa $(\mathrm{p}>0,05)$. 
Tabla 3: Resultados análisis de regresión logística

Reducción del presupuesto dedicado a las políticas de género

\begin{tabular}{|c|c|c|c|c|}
\hline & $B$ & S.E. & Sig. & $\operatorname{Exp}(B)$ \\
\hline \multicolumn{5}{|c|}{$\begin{array}{l}\text { Ideología del partido político en el gobierno } \\
\text { Partido de Centro Izuierda (Ref.) }\end{array}$} \\
\hline Partido político & & & 0,00 & \\
\hline Partido de Centro & 0,73 & 0,64 & 0,26 & 2,07 \\
\hline Partido de Centro-Derecha & 2,22 & 0,63 & 0,00 & 9,18 \\
\hline Constante & 0,06 & 0,35 & 0,86 & 1,06 \\
\hline
\end{tabular}

Se puede concluir, por lo tanto, que la variable crisis económica tiene una influencia determinante en los recortes que se están produciendo en los presupuestos dedicados a las políticas de género en España. No obstante, el hallazgo si cabe más relevante es que durante los periodos de crisis la ideología de los partidos políticos gobernantes se erige también en una variable explicativa de dichos recortes. Así, si bien en el periodo de bonanza las diferencias entre los partidos políticos no resultaban relevantes, durante la crisis dichas diferencias se vuelven significativas, de tal manera que es mucho más probable que se produzcan recortes en las políticas de género cuando están en el gobierno los partidos políticos ubicados ideológicamente en una posición de centro-derecha.

\subsection{Los retrocesos en el marco normativo: ¿son siempre feministas las políticas de igualdad?}

Si bien los recursos representan una dimensión fundamental para conocer el desarrollo de las políticas de género, existen otros elementos que configuran el núcleo de este tipo de actuaciones. Por lo que se refiere a sus principales instrumentos, las políticas de género en España comenzaron por situar a los planes plurianuales de igualdad como su principal herramienta de planificación (Astelarra, 2005; Bustelo, 2004). Sin embargo, durante la década de los 2000 otro instrumento clave irrumpe en esta área competencial (Bustelo, 2014). Se trata de las leyes de igualdad, que comienzan su andadura en 2002 con la aprobación en Navarra de la Ley Foral 33/2002, y que cuentan en la actualidad con doce ejemplos a nivel autonómico y con la Ley Orgánica 3/2007 a nivel estatal (Tabla 4). Aunque todas ellas incluyen el objetivo general de fomentar la igualdad entre mujeres y hombres, podemos establecer al menos dos grandes etapas en su desarrollo. Las primeras 
normas aprobadas, que se corresponden con autonomías gobernadas por ejecutivos conservadores (Navarra, Galicia, etc.), se proponen dos objetivos esenciales. Por una parte, proclaman la igualdad entre mujeres y hombres como un objetivo general de las administraciones públicas autonómicas. Por otra, establecen propósitos y medidas para áreas concretas como la educación, el empleo o la salud. La aprobación de la Ley 4/2005, para la igualdad entre mujeres y hombres de Euskadi marca sin embargo un cambio en la predominancia de dicho modelo. Las normas refrendadas a continuación ${ }^{5}$-incluyendo la Ley Orgánica 3/2007- reproducen de modo claro el esquema y los contenidos propuestos en la ley vasca, con lo que representan un salto cualitativo respecto a los primeros textos. Se incorpora por primera vez la definición de conceptos y principios clave tales como la igualdad de trato, la igualdad de oportunidades, etc., y se recoge la creación de amplios y ambiciosos mecanismos para incorporar la igualdad en el proceso político (presupuestos de género, unidades de igualdad etc.) e implementar así el denominado mainstreaming de género (Alonso, 2010). Se opta así por un concepto de igualdad amplio - no solo referido a la igualdad formal, no solo referido a las mujeres etc.-, y que implica la transformación del proceso de elaboración de las políticas públicas. En cualquier caso, y a pesar de la existencia de diferencias entre ambos periodos, la generalización de este tipo de normas ha puesto en evidencia la capacidad de emulación entre CCAA en lo que concierne a las políticas de igualdad y su consiguiente expansión a lo largo del tiempo (Alonso, 2015) ${ }^{6}$.

Si bien ha existido una clara propensión a regular la promoción de la igualdad, de modo que se ampliaran progresivamente los instrumentos y las áreas concernidas, los años más recientes, en concreto los correspondientes al periodo de crisis, han sido testigo de cambios relevantes en lo referido a esta dimensión. En primer lugar, se ha visto frenada la tendencia expansiva de este tipo de normas. De hecho, en los últimos años contamos con numerosos ejemplos de leyes de igualdad que se encontraban en proceso de tramitación y que debido a un cambio en la mayoría parlamentaria fueron finalmente rechazadas. El primer ejemplo de ello viene representado por la comunidad gallega, que celebró elecciones en el año 2009 y cuyo cambio de ejecutivo determinó no solo la eliminación de un organismo de igualdad, sino también la paralización de un borrador de ley que todavía estaba en una fase inicial. El cambio político en Cataluña, que tuvo lugar en el año 2010, significó la paralización del Proyecto de Ley para una nueva ciudadanía y para la

\footnotetext{
${ }^{5}$ Con la excepción de la Ley 12/2006 aprobada en Islas Baleares, que se inspira en mayor medida en el modelo precedente, y en especial en el proporcionado por la ley gallega de igualdad.

${ }^{6}$ Es necesario destacar que la lucha contra la violencia de género ha ocupado cada vez mayor protagonismo. Ha sido incorporada como ámbito de actuación en todas las leyes de igualdad y en muchos casos se han aprobado normas específicas al respecto.
} 
igualdad efectiva entre mujeres y hombres que no llegó a ser votado en el Parlamento autonómico debido a la disolución del mismo. Una situación similar tuvo lugar en Cantabria, que en 2011 también cambió el color de su ejecutivo con la entrada del Partido Popular (PP), paralizando la aprobación del que ya era el Proyecto de Ley de Cantabria para la Igualdad Efectiva entre Mujeres y Hombres ${ }^{7}$. En las Islas Baleares el Proyecto de ley de igualdad entre mujeres y hombres ${ }^{8}$ sí resultó votado por el Parlamento autonómico, aunque no obtuvo la mayoría necesaria. La presencia de un gobierno con minoría en la cámara, así como el voto en contra del PP, determinó el fracaso en la aprobación de una nueva ley balear en materia de igualdad ${ }^{9}$. Finalmente, la derrota electoral del PSOE a nivel estatal en 2011 ha significado la paralización del Proyecto de Ley integral para la igualdad de trato y la no discriminación, una norma que se centraba en desarrollar el derecho antidiscriminatorio en España.

Es necesario resaltar que la paralización de todas estas normas representa un claro retroceso en materia de políticas de igualdad, frenando la tendencia expansiva de las últimas décadas. A pesar de que esta coincide con el inicio del periodo de crisis económica, parece claro que se relaciona de modo notable con el cambio en el color político del gobierno, en concreto con la aparición de ejecutivos de centroderecha.

La regulación de un nuevo y controvertido ámbito de actuación resulta también reseñable a este respecto. Así, desde el año 2008 se han aprobado en España hasta 6 normas relativas a la protección de la mujer embarazada (Castilla y León, Murcia, Galicia, Castilla La Mancha, Comunidad de Madrid, La Rioja), mientras que dos nuevos textos han sido anunciados (Islas Baleares, Extremadura ${ }^{10}$ ) (Alonso, 2013). Con la excepción de la norma castellano manchega, todas ellas se corresponden con parlamentos de mayoría conservadora, en concreto del PP. Además, comparten el hecho de haber sido impulsadas por sendas Iniciativas Legislativas Populares auspiciadas por la Red Madre ${ }^{11}$, una fundación centrada en apoyar a las mujeres gestantes con dificultades y evitar la interrupción del embarazo.

7 Proyecto de Ley de Cantabria para la Igualdad Efectiva entre Mujeres y Hombres. 7L/1000-0026. BOPC núm. 476, de 15 de marzo de 2011.

${ }^{8}$ RGE núm. 3056/10, de igualdad de mujeres y hombres de las Islas Baleares. BOPIB núm. 137, de 16 de julio de 2010

${ }^{9}$ Esta norma ha sido presentada en la presente legislatura como proposición de ley y ha sido de nuevo rechazada por el Parlamento balear de mayoría popular.

${ }^{10}$ En Extremadura se ha llegado a aprobar el Plan de la Salud Reproductiva y Abordaje Específico del Embarazo, que cuenta con fines similares.

${ }^{11}$ Véase http://www.redmadre.es/. 
El interés de esta normativa reside en el giro copernicano que representa respecto a las leyes de igualdad precedentes. Estas nuevas normas se enmarcan en la voluntad de promover la protección de la maternidad y el apoyo a las mujeres gestantes. En general, entienden que las mujeres que se encuentran en situación de vulnerabilidad (menores, mujeres sin recursos, migrantes etc.) sufren situaciones de desigualdad que determinan que en muchas ocasiones deban renunciar a la maternidad. Este objetivo general aborda uno de los ámbitos tradicionales de las políticas de igualdad, como es la conciliación entre la vida personal y laboral. Con ello parece otorgar continuidad al marco normativo existente. No obstante, un rápido repaso al contenido de las nuevas leyes, en concreto a aquellas aprobadas por el PP, remite a un cambio radical de perspectiva ${ }^{12}$. En primer lugar, dichas normas centran su atención en un propósito central: la culminación de la gestación. Mientras algunos textos expresan de modo explícito su propósito de proteger el derecho a la vida desde la concepción (Madrid, Galicia), otros enfatizan que el aborto representa una tragedia tanto para la madre como para el no nacido (Castilla y León, Murcia, La Rioja). Con ello ponen de relieve que las actuaciones públicas han de dirigirse a evitar la interrupción del embarazo. Así lo indican las medidas recogidas en las diferentes leyes, las cuales consisten en esencia en proveer a las mujeres gestantes de información y asesoramiento acerca de los recursos existentes para el apoyo a la maternidad, o en su caso, acerca de aquellos previstos para los procesos de adopción y acogimiento ${ }^{13}$. La apuesta por la protección del derecho a la vida determina, de este modo, que se centre la atención en las mujeres embarazadas y en evitar que se produzca un aborto. Este enfoque representa un claro cambio respecto a las políticas anteriores, tanto en lo referido a la conciliación como a la interrupción voluntaria del embarazo. Así, se opta por políticas que no abarcan a todas las mujeres, ya que se focalizan en aquellas que ya están embarazadas, y que no contemplan de modo claro la autonomía de las mismas para decidir sobre su propia maternidad, obviando aquellos recursos existentes en materia de interrupción de la gestación.

En esta misma línea, el papel otorgado a los hombres en estas nuevas leyes autonómicas cuestiona igualmente su coherencia con el marco normativo existente.

\footnotetext{
${ }^{12}$ La norma aprobada en Castilla La Mancha se dirige tanto a la protección de la mujer embarazada como de las familias numerosas. No incorpora referencias relativas a la protección de la vida y del no nacido y otorga un papel más relevante a la paternidad. Con ello se distancia del modelo propuesto por las leyes surgidas en comunidades gobernadas por el PP.

${ }^{13}$ De hecho todas las normas dedican gran parte de su articulado a prever la creación de una red de apoyo a la mujer embarazada. Para ello contemplan subvencionar a entidades sin ánimo de lucro análogas a la Red Madre, aquella que impulsó inicialmente este tipo de normas.
} 
De este modo, las políticas de género habían evolucionado hacia un concepto de igualdad de naturaleza relacional, que implicaba en mayor medida la transformación de los roles de ambos sexos (Bustelo y Lombardo, 2007). Las normas relativas a la protección de la mujer embarazada focalizan, sin embargo, toda su atención en el rol de las mujeres como madres, mientras que los varones están prácticamente ausentes, y las escasas medidas propuestas se limitan a fomentar la sensibilización respecto al valor de la paternidad.

La confluencia de todos estos elementos hace que resulte pertinente recuperar algunas de las preguntas clave de aquella literatura en materia de políticas de género que centra su atención en el contenido de las mismas. Así se muestra adecuado 'revisitar' cuestiones como las siguientes: ¿qué entendemos por igualdad entre mujeres y hombres? ¿Son siempre transformadoras aquellas políticas que pretenden promoverla? ¿Pueden contener los mismos estereotipos que deberían eliminar? (Verloo, 2007) Desde esta perspectiva, esta normativa recientemente aprobada puede ser concebida en términos de retroceso para las políticas de género, dado que cuestiona la autonomía de las mujeres, las limita a su rol de madres y obvia la necesaria transformación de los roles de los hombres. Con ello, nos sitúa ante un concepto de igualdad que reproduce algunos de los estereotipos que tradicionalmente las políticas de género habían intentado subvertir.

Paralelamente, el análisis de estas normas permite explorar de nuevo la incidencia de la variable 'ideología del partido político en el gobierno' a partir de otra dimensión clave como es la legislación. Así, resulta evidente que estas nuevas leyes están vinculadas de modo claro a la agenda del Partido Popular en materia de salud sexual y reproductiva. En este sentido, van en coherencia con propuestas centrales como la reforma de la ley del aborto, retirada en 2014, que pretendía limitar la capacidad de las mujeres de decidir libremente sobre la interrupción de su embarazo y que se enmarcaba en su apuesta general por la protección del derecho a la vida ${ }^{14}$. El Anteproyecto de Ley Orgánica para la Protección de la Vida del Concebido y de los Derechos de la Mujer Embarazada determinaba de hecho que se había de establecer un sistema legal para la defensa del nasciturus, como bien jurídicamente protegido. Para ello proponía autorizar la interrupción del embarazo solamente en unos supuestos muy determinados -violación o grave peligro para la salud física o psíquica de la madre-. A su vez, y en línea con las normas

\footnotetext{
${ }^{14}$ El programa electoral para los comicios generales de 2011 indicaba que el PP cambiaría el modelo de la actual regulación sobre el aborto para reforzar la protección del derecho a la vida. Igualmente establecía que se aprobarían leyes de apoyo a la mujer embarazada en los niveles estatal y autonómico.
} 
autonómicas, proponía reforzar los sistemas de información orientados a ofrecer alternativas a la práctica del aborto y a advertir de sus riesgos para la salud de la mujer.

Este marco normativo difiere en gran medida de los posicionamientos del movimiento feminista y de mujeres que tradicionalmente ha puesto el énfasis en la autonomía de estas para decidir sobre su maternidad y para disponer libremente de su propio cuerpo (Stetson, 2001). En este sentido, constituye un cambio notable en la agenda relativa a la igualdad, cambio que solamente puede ser concebido en términos de claro retroceso.

Tabla 4. Leyes de igualdad y leyes de apoyo a la mujer embarazada aprobadas en España (2002-2012).

COMUNIDAD AUTÓNOMA

Andalucía Ley 12/2007, de 26 de noviembre, para la promoción de la igualdad de género en

Andalucía

\begin{tabular}{|c|c|c|}
\hline Andalucía & $\begin{array}{c}\text { Ley } 12 / 2007 \text {, de } 26 \text { de noviembre, para la } \\
\text { promoción de la igualdad de género en } \\
\text { Andalucía }\end{array}$ & \\
\hline Asturias & $\begin{array}{c}\text { Ley del Principado de Asturias } 2 / 2011 \text {, de } \\
11 \text { de marzo, para la igualdad de mujeres } \\
\text { y hombres y la erradicación de la } \\
\text { violencia de género }\end{array}$ & \\
\hline Baleares & $\begin{array}{c}\text { Ley } 12 / 2006 \text {, de } 20 \text { de septiembre, para la } \\
\text { mujer }\end{array}$ & En proceso de elaboración \\
\hline Canarias & $\begin{array}{l}\text { Ley } 1 / 2010, \text { de } 26 \text { de febrero, canaria de } \\
\text { igualdad entre mujeres y hombres }\end{array}$ & \\
\hline $\begin{array}{c}\text { Castilla la } \\
\text { Mancha }\end{array}$ & $\begin{array}{l}\text { Ley } 12 / 2010 \text {, de } 18 \text { de noviembre, de } \\
\text { igualdad entre mujeres y hombres de } \\
\text { Castilla-La Mancha. }\end{array}$ & $\begin{array}{c}\text { Ley } 17 / 2010 \text {, de } 29 \text { de diciembre, de las } \\
\text { familias numerosas de Castilla-La } \\
\text { Mancha y de la maternidad }\end{array}$ \\
\hline Castilla y León & $\begin{array}{c}\text { Ley } 1 / 2003 \text {, de } 3 \text { marzo } 2003 \text {, de Igualdad } \\
\text { de Oportunidades entre Mujeres y } \\
\text { Hombres en Castilla y León }\end{array}$ & $\begin{array}{c}\text { Ley } 14 / 2008 \text {, de } 18 \text { de diciembre, por la } \\
\text { que se crea y regula una red de apoyo a la } \\
\text { mujer embarazada }\end{array}$ \\
\hline Extremadura & $\begin{array}{c}\text { Ley } 8 / 2011 \text {, de } 23 \text { de marzo, de Igualdad } \\
\text { entre Mujeres y Hombres y contra la } \\
\text { Violencia de Género en Extremadura }\end{array}$ & En proceso de elaboración \\
\hline \multirow[t]{2}{*}{ Galicia } & $\begin{array}{l}\text { Ley } 7 / 2004 \text {, de } 16 \text { de julio, gallega para la } \\
\text { igualdad de mujeres y hombres }\end{array}$ & $\begin{array}{c}\text { Ley } 5 / 2010 \text {, de } 23 \text { de junio, por la que se } \\
\text { establece y regula una red de apoyo a la } \\
\text { mujer embarazada }\end{array}$ \\
\hline & $\begin{array}{c}\text { Ley } 2 / 2007 \text {, de } 28 \text { de marzo, del trabajo } \\
\text { en igualdad de las mujeres de Galicia }\end{array}$ & \\
\hline La Rioja & & Ley $1 / 2012$, de 12 de junio, por la que se \\
\hline
\end{tabular}

\begin{tabular}{|c|c|c|}
\hline Andalucía & $\begin{array}{c}\text { Ley } 12 / 2007 \text {, de } 26 \text { de noviembre, para la } \\
\text { promoción de la igualdad de género en } \\
\text { Andalucía }\end{array}$ & \\
\hline Asturias & $\begin{array}{c}\text { Ley del Principado de Asturias } 2 / 2011 \text {, de } \\
11 \text { de marzo, para la igualdad de mujeres } \\
\text { y hombres y la erradicación de la } \\
\text { violencia de género }\end{array}$ & \\
\hline Baleares & $\begin{array}{c}\text { Ley } 12 / 2006 \text {, de } 20 \text { de septiembre, para la } \\
\text { mujer }\end{array}$ & En proceso de elaboración \\
\hline Canarias & $\begin{array}{l}\text { Ley } 1 / 2010, \text { de } 26 \text { de febrero, canaria de } \\
\text { igualdad entre mujeres y hombres }\end{array}$ & \\
\hline $\begin{array}{c}\text { Castilla la } \\
\text { Mancha }\end{array}$ & $\begin{array}{l}\text { Ley } 12 / 2010 \text {, de } 18 \text { de noviembre, de } \\
\text { igualdad entre mujeres y hombres de } \\
\text { Castilla-La Mancha. }\end{array}$ & $\begin{array}{c}\text { Ley } 17 / 2010 \text {, de } 29 \text { de diciembre, de las } \\
\text { familias numerosas de Castilla-La } \\
\text { Mancha y de la maternidad }\end{array}$ \\
\hline Castilla y León & $\begin{array}{c}\text { Ley } 1 / 2003 \text {, de } 3 \text { marzo } 2003 \text {, de Igualdad } \\
\text { de Oportunidades entre Mujeres y } \\
\text { Hombres en Castilla y León }\end{array}$ & $\begin{array}{c}\text { Ley } 14 / 2008 \text {, de } 18 \text { de diciembre, por la } \\
\text { que se crea y regula una red de apoyo a la } \\
\text { mujer embarazada }\end{array}$ \\
\hline Extremadura & $\begin{array}{c}\text { Ley } 8 / 2011 \text {, de } 23 \text { de marzo, de Igualdad } \\
\text { entre Mujeres y Hombres y contra la } \\
\text { Violencia de Género en Extremadura }\end{array}$ & En proceso de elaboración \\
\hline \multirow[t]{2}{*}{ Galicia } & $\begin{array}{l}\text { Ley } 7 / 2004 \text {, de } 16 \text { de julio, gallega para la } \\
\text { igualdad de mujeres y hombres }\end{array}$ & $\begin{array}{c}\text { Ley } 5 / 2010 \text {, de } 23 \text { de junio, por la que se } \\
\text { establece y regula una red de apoyo a la } \\
\text { mujer embarazada }\end{array}$ \\
\hline & $\begin{array}{c}\text { Ley } 2 / 2007 \text {, de } 28 \text { de marzo, del trabajo } \\
\text { en igualdad de las mujeres de Galicia }\end{array}$ & \\
\hline La Rioja & & Ley $1 / 2012$, de 12 de junio, por la que se \\
\hline
\end{tabular}

\begin{tabular}{|c|c|c|}
\hline Andalucía & $\begin{array}{c}\text { Ley } 12 / 2007 \text {, de } 26 \text { de noviembre, para la } \\
\text { promoción de la igualdad de género en } \\
\text { Andalucía }\end{array}$ & \\
\hline Asturias & $\begin{array}{c}\text { Ley del Principado de Asturias } 2 / 2011 \text {, de } \\
11 \text { de marzo, para la igualdad de mujeres } \\
\text { y hombres y la erradicación de la } \\
\text { violencia de género }\end{array}$ & \\
\hline Baleares & $\begin{array}{c}\text { Ley } 12 / 2006 \text {, de } 20 \text { de septiembre, para la } \\
\text { mujer }\end{array}$ & En proceso de elaboración \\
\hline Canarias & $\begin{array}{l}\text { Ley } 1 / 2010, \text { de } 26 \text { de febrero, canaria de } \\
\text { igualdad entre mujeres y hombres }\end{array}$ & \\
\hline $\begin{array}{c}\text { Castilla la } \\
\text { Mancha }\end{array}$ & $\begin{array}{l}\text { Ley } 12 / 2010 \text {, de } 18 \text { de noviembre, de } \\
\text { igualdad entre mujeres y hombres de } \\
\text { Castilla-La Mancha. }\end{array}$ & $\begin{array}{c}\text { Ley } 17 / 2010 \text {, de } 29 \text { de diciembre, de las } \\
\text { familias numerosas de Castilla-La } \\
\text { Mancha y de la maternidad }\end{array}$ \\
\hline Castilla y León & $\begin{array}{c}\text { Ley } 1 / 2003 \text {, de } 3 \text { marzo } 2003 \text {, de Igualdad } \\
\text { de Oportunidades entre Mujeres y } \\
\text { Hombres en Castilla y León }\end{array}$ & $\begin{array}{c}\text { Ley } 14 / 2008 \text {, de } 18 \text { de diciembre, por la } \\
\text { que se crea y regula una red de apoyo a la } \\
\text { mujer embarazada }\end{array}$ \\
\hline Extremadura & $\begin{array}{c}\text { Ley } 8 / 2011 \text {, de } 23 \text { de marzo, de Igualdad } \\
\text { entre Mujeres y Hombres y contra la } \\
\text { Violencia de Género en Extremadura }\end{array}$ & En proceso de elaboración \\
\hline \multirow[t]{2}{*}{ Galicia } & $\begin{array}{l}\text { Ley } 7 / 2004 \text {, de } 16 \text { de julio, gallega para la } \\
\text { igualdad de mujeres y hombres }\end{array}$ & $\begin{array}{c}\text { Ley } 5 / 2010 \text {, de } 23 \text { de junio, por la que se } \\
\text { establece y regula una red de apoyo a la } \\
\text { mujer embarazada }\end{array}$ \\
\hline & $\begin{array}{c}\text { Ley } 2 / 2007 \text {, de } 28 \text { de marzo, del trabajo } \\
\text { en igualdad de las mujeres de Galicia }\end{array}$ & \\
\hline La Rioja & & Ley $1 / 2012$, de 12 de junio, por la que se \\
\hline
\end{tabular}

En proceso de elaboración

Galicia Ley $7 / 2004$, de 16 de julio, gallega para la igualdad de mujeres y hombres

Ley $2 / 2007$, de 28 de marzo, del trabajo en igualdad de las mujeres de Galicia 


\begin{tabular}{|c|c|c|}
\hline & & $\begin{array}{l}\text { establece y regula una red de apoyo a la } \\
\text { mujer embarazada }\end{array}$ \\
\hline Madrid & & $\begin{array}{c}\text { Ley } 3 / 2011 \text {, de } 22 \text { de marzo, por la que } \\
\text { se establece y regula una red de apoyo a } \\
\text { la mujer embarazada }\end{array}$ \\
\hline Murcia & $\begin{array}{c}\text { Ley } 7 / 2007 \text {, de } 4 \text { de abril, para la Igualdad } \\
\text { entre Mujeres y Hombres, y de Protección } \\
\text { contra la Violencia de Género en la } \\
\text { Región de Murcia }\end{array}$ & $\begin{array}{c}\text { Ley } 11 / 2009 \text {, de } 1 \text { de diciembre, por la } \\
\text { que se establece y regula una Red de } \\
\text { Apoyo a la Mujer Embarazada }\end{array}$ \\
\hline Navarra & $\begin{array}{l}\text { Ley foral } 33 / 2002 \text {, de } 28 \text { de noviembre, de } \\
\text { fomento de la igualdad de oportunidades } \\
\text { entre mujeres y hombres de Navarra }\end{array}$ & \\
\hline País Vasco & $\begin{array}{l}\text { Ley } 4 / 2005 \text { para la igualdad entre mujeres } \\
\text { y hombres }\end{array}$ & \\
\hline Valencia & $\begin{array}{c}\text { Ley } 9 / 2003 \text {, de } 2 \text { de abril, de la } \\
\text { Generalitat, para la Igualdad entre } \\
\text { Mujeres y Hombres. }\end{array}$ & \\
\hline España & $\begin{array}{c}\text { Ley orgánica } 3 / 2007 \text {, de } 22 \text { de marzo, } \\
\text { para la igualdad efectiva de mujeres y } \\
\text { hombres. }\end{array}$ & $\begin{array}{c}\text { Anteproyecto de Ley Orgánica para la } \\
\text { Protección de la Vida del Concebido y de } \\
\text { los Derechos de la Mujer Embarazada } \\
\text { (retirado) }\end{array}$ \\
\hline
\end{tabular}

Fuente: elaboración propia

\subsection{El cuestionamiento de la arquitectura institucional}

Otra de las dimensiones centrales de las políticas de género viene representada por los organismos de igualdad. Por una parte, múltiples estudios los han considerado como una variable dependiente de especial interés, entendiendo, por ejemplo, que la creación de este tipo de instituciones marcaba el nacimiento de una nueva área competencial (Bustelo, 2004; Valiente, 1994). En otros casos, se ha concebido al organismo de igualdad como el factor explicativo de la presencia de avances respecto a otro tipo de factores (aprobación de legislación, representación descriptiva etc.) (McBride y Mazur, 2010). En este estudio apostamos por explorar su presencia como una dimensión de las políticas de igualdad que nos permite medir y explicar los retrocesos en este ámbito.

En el caso de España, la creación de este tipo de entidades marcó sin duda el surgimiento de las políticas de género. El Instituto de la Mujer del nivel estatal fue 
pionero a este respecto con su aparición en $1983^{15}$ y marcó la pauta para la progresiva extensión de su modelo al nivel autonómico. De hecho, en 1988 surgen ya organismos autónomos análogos en Andalucía y Euskadi y progresivamente se incorporan a la arquitectura institucional de gran parte de las CCAA ${ }^{16}$. En 2006, cuando se crea la última entidad de estas características en Navarra, existían ya en un total de 12 autonomías. Las restantes habían previsto igualmente la creación de estructuras para la promoción de la igualdad, pero en este caso adoptaban fórmulas diversas tales como direcciones generales o secretarías. En cualquier caso, las últimas décadas fueron testigo de la expansión y consolidación del denominado feminismo de Estado, de modo que todos los niveles de gobierno, incluyendo el local (Paleo, 2011), apostaron por ampliar su arquitectura institucional con el objeto de promover la igualdad de género (Bustelo, 2004).

Esta tendencia positiva se ha visto interrumpida en los años más recientes, en concreto desde el inicio del periodo de crisis económica. De hecho, contamos ya con diversos e ilustrativos ejemplos de eliminación de estructuras de este tipo al amparo de proyectos de 'racionalización' de la Administración Pública. El Servizo Galego de Igualdade, por ejemplo, fue eliminado en el año 2010 por el gobierno de Núñez Feijoo después de 19 años de historia, de modo que todas las competencias en materia de igualdad resultaron concentradas en la Secretaría General de Igualdad creada en el año 2005. La eliminación de este organismo autónomo se justificó no solo en la consecución de "una mayor eficiencia y eficacia en la aplicación de las políticas de igualdad", sino también en que "esta nueva estructura servirá para aplicar con mayor eficacia la transversalidad del principio de igualdad en todas las políticas y acciones desarrolladas por la Xunta de Galicia” ${ }^{, 17}$. El mainstreaming sirvió, de este modo, junto con los valores de eficacia, eficiencia y racionalización del sector público, como elemento justificativo de la disminución de los recursos dedicados a la promoción de la igualdad. En este caso,

15 Existieron entidades de menor relevancia con anterioridad. Ya en 1977 el gobierno de UCD creó una primera estructura, la Subdirección General de la Condición Femenina, que se ocupó de comenzar a revisar aquella legislación que discriminaba de modo abierto a las mujeres y de crear los primeros centros de información en ciertas ciudades. Dicho organismo pasa a denominarse Subdirección General de la Mujer en 1980, aunque continúa teniendo un limitado peso organizativo en el ejecutivo.

16 Existían ejemplos previos de organismos menores tales como la Comisión Interdepartamental de la Mujer de Andalucía creada en 1983 o la instituida en Cataluña en 1987.

${ }^{17}$ Ley 7/2010, de 15 de octubre, por la que se suprime el organismo autónomo Servicio Gallego de Promoción de la Igualdad del Hombre y la Mujer y se modifican determinados artículos de la Ley 2/2007, de 28 de marzo, del trabajo en igualdad de las mujeres de Galicia 
mientras se eliminaba un organismo autónomo con personalidad jurídica propia y una larga trayectoria, se mantenía una estructura departamental con un menor grado de independencia. El ejemplo gallego coincide así con aquellos análisis que han mostrado como el desmantelamiento de organismos de igualdad se ha visto justificado en el desarrollo del mainstreaming y en su carácter transversal, no vinculado a ningún área de gobierno (Stratigaki, 2005).

El contexto de crisis económica y financiera, así como la necesidad de 'racionalizar' el funcionamiento y tamaño del sector público constituyen también las razones aludidas por la Ley 9/2010 aprobada en la Comunidad de Madrid que elimina su Consejo de la Mujer creado en 1993 y que disminuye de este modo las vías de participación existentes para el movimiento de mujeres ${ }^{18}$. El Instituto de la Mujer de la Región de Murcia, por su parte, fue eliminado por el ejecutivo popular en el año 2010 argumentando que ya existían otras áreas o departamentos que cumplían con funciones análogas y que no era necesario mantener un organismo autónomo para dicho fin ${ }^{19}$. Han desaparecido también entidades pioneras como la Unidad de Género del gobierno cántabro, que ocupaba un rol central en la promoción de la transversalidad en la Administración autonómica.

A pesar de que estas importantes eliminaciones coinciden con la presencia o aparición de ejecutivos conservadores, existen también ejemplos de retrocesos propiciados por gobiernos socialistas. Así, no podemos obviar la eliminación del primer Ministerio de Igualdad que ha existido en España acontecida en 2010. Esta significó un evidente retroceso en la situación de la igualdad como una de las prioridades de la agenda política del gobierno de José Luís Rodríguez Zapatero. Así, las competencias en la materia pasaron de nuevo a formar parte de una Secretaría de Estado, un organismo de menor rango, y a situarse en un área competencial tradicionalmente vinculada a las políticas de igualdad, pero que carece de una posición central en el organigrama de gobierno, como es el Ministerio de Sanidad ${ }^{20}$.

Todos estos ejemplos muestran, por lo tanto, el cuestionamiento de uno de los elementos centrales de las políticas de género, el cual se había visto

${ }^{18}$ Ley 9/2010 de Medidas Fiscales Administrativas y de Racionalización del Sector Público.

${ }^{19}$ Decreto 246/2010, de 10 de septiembre, de Extinción del Organismo Autónomo Instituto de la Mujer de la Región de Murcia.

${ }^{20}$ El nivel jerárquico y/o el área competencial a la que se adscribe el organismo de igualdad puede resultar también una dimensión de interés para futuras investigaciones. 
ampliamente consolidado en las últimas décadas. Aunque la crisis económica y la consiguiente necesidad de racionalizar el gasto público se aducen como principales argumentos para suprimir los organismos de igualdad, los ilustrativos ejemplos existentes invitan de nuevo a explorar la incidencia de la variable del partido político, dado que son los gobiernos conservadores los que con mayor frecuencia han optado por la eliminación de dichas entidades.

\section{CONCLUSIONES}

Este trabajo se enmarca en aquellas agendas de investigación relativas a las políticas de género y a las variables que explican su desarrollo. Sin embargo, se ha apostado por emplear una novedosa perspectiva de análisis centrada en la identificación de los retrocesos acontecidos desde el inicio de la crisis económicofinanciera y en la valoración de si este acontecimiento constituye la única causa de los mismos. El caso español ha representado un objeto de análisis de especial interés. Así, las políticas de género habían experimentado una clara expansión durante las últimas décadas, adquiriendo un notable carácter multinivel. Desde el inicio de la crisis y de las consiguientes políticas de austeridad, no obstante, se han producido reseñables retrocesos que han afectado al núcleo de esta área competencial. En este capítulo hemos identificado este tipo de involuciones en lo relativo a tres dimensiones clave: los recursos económicos destinados a la promoción de la igualdad, los cuales han disminuido claramente en los últimos años; la normativa específica en este ámbito, que o bien ha visto paralizado su proceso de aprobación o bien ha reorientado su contenido; y finalmente los organismos de igualdad, principales valedores del impulso de esta área de gobierno, y que han sido ampliamente cuestionados.

El interés de este trabajo reside además en su voluntad de determinar los factores que explican este tipo de retrocesos. En concreto centra su atención en explorar dos variables que parecen ganar protagonismo durante el periodo en el que se producen los mismos. Así, no resulta posible obviar el análisis de la variable 'crisis económica', que sin duda marca el devenir de las políticas públicas españolas en los últimos años. Tampoco se podía desdeñar la influencia de la variable 'ideología del partido político', que no solo ha sido destacada como relevante por parte de la literatura especializada, sino que ha ganado pertinencia con la presencia de múltiples cambios en los ejecutivos de los niveles estatal y autonómico.

En efecto, las tres dimensiones analizadas arrojan conclusiones de interés respecto a ambas variables. Por lo que respecta a la dimensión referida a los presupuestos, tal como era de esperar, se observa que la crisis económica tiene una influencia determinante en los recortes que se están produciendo en ambos niveles de gobierno. De hecho, la disminución de recursos producida en los últimos años se 
muestra muy notable. No obstante, la conclusión si cabe más relevante de este análisis indica que durante los periodos de crisis, la ideología de los partidos políticos gobernantes se erige también en una variable explicativa de dichos recortes. Así, si bien durante la época de bonanza las diferencias entre los partidos políticos en relación a esta dimensión no resultaban relevantes, durante el periodo de crisis estas se vuelven significativas, de tal manera que es mucho más probable que se produzcan recortes en las políticas de género cuando están en el gobierno los partidos políticos ubicados ideológicamente en una posición de centro-derecha.

La dimensión referida a la legislación en materia de igualdad resulta igualmente reveladora. De hecho, se observa de modo claro que en los últimos años, coincidiendo con el inicio de la crisis económica, se han producido retrocesos considerables. Por una parte, diversos proyectos de ley se han visto paralizados, determinando un cambio de tendencia respecto a la progresiva ampliación del marco normativo en materia de igualdad. Por otra parte, ha emergido un nuevo ámbito regulativo, como es el referente a la protección de la mujer embarazada, que ha implicado un cambio sustancial en el enfoque respecto a leyes precedentes. Estos retrocesos muestran de nuevo una clara coincidencia con la presencia de partidos de centro-derecha en el poder. En este sentido, su entrada o permanencia en el gobierno guarda evidente relación con la paralización de nuevas leyes de igualdad y con la introducción de aquellas relativas al apoyo a las mujeres gestantes.

Un proceso similar acontece en lo respectivo a los organismos de igualdad. Los notables ejemplos de eliminación de este tipo de estructuras ocurren con el inicio de la crisis económica y se justifican en base a la necesidad de racionalizar la estructura de la Administración Pública y de incrementar su eficacia y eficiencia. Sin embargo, son de nuevo los partidos conservadores los que con mayor frecuencia optan por eliminar un recurso tan central para las políticas de género como son los organismos de igualdad.

Del análisis de estas tres dimensiones se puede concluir, por lo tanto, la relevancia de ambas variables para entender los notables retrocesos sufridos por esa área competencial. La crisis económico-financiera parece representar el contexto que justifica la necesidad de frenar la expansión de las actuaciones en materia de igualdad. De hecho, puede ser concebida como la ventana de oportunidad que permite poner en cuestión elementos ampliamente consolidados tales como los analizados en este trabajo. Sin embargo, la variable ideología del partido político arroja también interesantes conclusiones a este respecto. Si bien se observa que los retrocesos coinciden con el periodo de crisis, se pone en evidencia que estos se producen con mucha mayor intensidad cuando existe un gobierno de centro o centro-derecha. 
Estas conclusiones ponen de manifiesto una vez más la necesidad de explorar las políticas de género a través de factores tanto relacionados con la agencia como con la estructura. Igualmente, revelan la pertinencia de centrar la atención de modo especial en la presencia de retrocesos en esta área de gobierno. También de escudriñar qué efectos ha tenido la crisis económica sobre las mismas y en qué medida los gobiernos conservadores han visto en esta la oportunidad para poner en cuestión los avances conseguidos hasta la fecha.

\section{BIBLIOGRAFÍA}

ALONSO, ALBA (2010): Las políticas de igualdad en España: construyendo el liderazgo del nivel subnacional. Administración \& Desarrollo, 38(52), 57-69

ALONSO, ALBA (2013): Retrocesos en materia de politicas de igualdade en España: Organismos, instrumentos e axenda política. En González, A., E. Aguayo, A. Jesús López eds., Roles de Xénero en tempos de Crise, 61-68, Santiago de Compostela: USC

ALONSO, ALBA (2015): El mainstreaming de género en España. Valencia: Tirant lo Blanch (en prensa)

ASTELARRA, JUDITH (2006): Veinte años de feminismo y políticas de equidad en España: 1980-2000. En Bosch, E. et al. comp., Los feminismos como herramientas de cambio social (I): Mujeres tejiendo redes históricas, desarrollos en el espacio público y estudios de las mujeres, Palma de Mallorca: UIB

ASTELARRA, JUDITH (2005): Veinte años de políticas de igualdad. Madrid: Cátedra.

BETTIO, FRANCESCA, MARCELLA CORSI, CARLO D'IPPOLITI, ANTIGONE LYBERAKI, MANUELA SAMEK Y ALINA VERASHCHAGINA (2012): The Impact of the Economic Crisis on the Situation of Women and Men and on Gender Equality Polices. Luxemburgo: European Commission

BUSTELO, MARÍA (2004): La evaluación de las políticas de igualdad de género, Madrid: Catarata

BUSTELO, MARÍA (2014): Three decades of state feminism and gender equality policies in multi-governed Spain, Sex Roles, vol. 70 (9/10), publicado online 15/06/2014 (DOI 10.1007/s11199-014-0381-9)

BUSTELO MARÍA y CANDICE ORTBALS (2007): The Evolution of Spanish State Feminism: a Fragmented Landscape. En Outshroorn, J. y Kantola, J. (eds.), Changing State Feminism, Basingstoke: Palgrave Macmillan

BUSTELO, MARÍA y EMANUELA LOMBARDO eds. (2007): Políticas de igualdad en España y en Europa. Madrid: Ed. Cátedra 
CHAPPEL, LOUISE (2000): Interacting with the State: Feminist Strategies and Political Opportunities. International Feminist Journal of Politics, 2(2), 244275

CHILDS, SARAH y MONA LENA KROOK (2009): Analysing Women's Substantive Representation: From Critical Mass to Critical Actors. Government and Opposition, 44(2), 125-145, DOI: 10.1111/j.1467-9248.2007.00712.x

DE VILLOTA, PALOMA (2011): El impacto de la crisis económica en los tributos y en la sostenibilidad del nuevo modelo social en España: Aproximación desde una perspectiva de género. Investigaciones feministas: papeles de estudios de mujeres, feministas y de género, 2, 71-94

EUROPEAN WOMEN'S LOBBY (2009): Women and the Economic Crisis- An opportunity to assert another vision of the world?. Bruselas, www.womenslobby.org, 09-05-2013

EUROPEAN WOMEN'S LOBBY (2012): The Price of Austerity: The Impact on Women's Rights and Gender Equality in Europe. Bruselas: EWL

GÁLVEZ, LINA (2012): La brecha de género en la crisis económica. Revista USTEA, Marzo, 2012.

GÁLVEZ, LINA y PAULA RODRÍGUEZ (2013): La desigualad de género en las crisis económica. Materials ICPS $\mathrm{n}^{\circ} 7$, Institut de Ciencias Polítiques i Socials, http://www.icps.cat/archivos/CiPdigital/CiP-I7Galvez Rodriguez.pdf, 09-052013

GONZÁLEZ, ELVIRA Y MARCELO SEGALES (2013): Women, gender equality and the economic crisis in Spain. En Karamessini, M. y Rubery, J. eds., Women and Austerity. The economic crisis and the future for gender equality, New York: Routledge

HANKIVSKI, OLENA (2008): Gender mainstreaming in Canada and Australia: A comparative analysis. Policy and Society, 27, 69-81, DOI: 10.1016/j.polsoc.2008.07.006

HAUSSMAN, MELISSA, MARIAN SAWER Y JILL VICKERS eds. (2010): Federalism, Feminism and Multilevel Governance. Burlington: Ashgate

HARCOURT, WENDY (2009): El impacto de la crisis en las mujeres de Europa Occidental. Brief 7, Asociación para los derechos de la Mujer y el Desarrollo (AWID),

http://www.awid.org/esl/content/download/101347/1181987/file/Brief\%207\%2 0Europa\%20Occidental.pdf., 10-20-2013

KARAMESSINI, MARIA (2013): Introduction: Women's vulnerability to recession and austerity. En Karamessini, M. y Rubery, J. eds., Women and Austerity. The Economic Crisis and the Future of Gender Equality, London: Routledge

KARAMESSINI, MARIA y JILL RUBERY (2013): Women and Austerity. The Economic Crisis and the Future of Gender Equality. London: Routledgde

KING, RICHARD y CAROLINE SWEETMAN (2010): Perspectivas de género sobre la crisis económica global. Documento de Trabajo de Oxfam Internacional. 
KROOK, MONA LENA y FIONA MACKAY eds. (2011): Gender, Politics and Institutions. Towards a Feminist Institutionalism. Nueva York: Palgrave

LANG, SABINE y BIRGIT SAUER (2012): Does Federalism Impact Gender Architectures? The Case of Women's Policy Agencies in Germany and Austria. Publius: The Journal of Federalism, 43(1), 68-89, DOI: 10.1093/publius/pjs048

LINOS, KATHERINA (2003): Are socialists a woman's best friend? Equality policies in the Spanish regions. Journal of European Public Policy, 10(3), 438 - 462, DOI: $10.1080 / 1350176032000085397$

LOMBARDO, EMANUELA y MAXIME FOREST eds. (2012): The Europeanization of gender equality policies. A sociological and discursive approach. Basingstoke: Palgrave McMillan

MAZUR, AMY (2003): Drawing Comparative Lessons from France and Germany. Review of Policy Research, 20(3), 493-523

McBRIDE, DOROTHY E. y AMY MAZUR (2010): The Politics of State Feminism. Innovations in Comparative Research. Philadelphia: Temple University Press

ORTBALS, CANDICE D. (2008): Feminized Decentralization. Evaluating Women's Representation in Spain. En Field, B. N. y Hamann, K. eds., Democracy and Institutional Development. Spain in Comparative Theoretical Perspective. Basingstoke: Palgrave Macmillan

PALEO, NATALIA (2011): Unha aproximación á análise das políticas municipais de xénero en Espana. Administración \& Cidadanía, 6(1), 91-107

PALEO, NATALIA (2013): A representación descriptiva das mulleres e as políticas de xénero. En González, A., E. Aguayo, A. Jesús López eds., Roles de Xénero en tempos de Crise, 69-76, Santiago de Compostela: USC

SQUIRES, JUDITH (2007): The new politics of gender equality. New York: Palgrave Macmillan

STETSON, DOROTHY M. ed. (2001): Abortion politics, women's movements, and the democratic state: a comparative study of state feminism. Oxford: Oxford University Press

SUBIRATS, JOAN, FREDERIC VARONE Y CORINNE LARRUE (2008): Análisis y gestión de políticas públicas. Barcelona: Editorial Ariel

STRATIGAKI, MARIA (2005): Gender Mainstreaming vs Positive Action: An Ongoing Conflict in EU Gender Equality Policy. European Journal of Women's Studies, 12(2), 165-186, DOI: 10.1177/1350506805051236

THRELFALL, MONICA (2007): Explaining Gender Parity Representation in Spain: The Internal Dynamics of Parties. West European Politics, 30(5), 1068-1095

VALIENTE, CELIA (2007): Are Gender Equality Institutions the Policy Allies of the Feminist Movement? A Contingent 'Yes' in the Spanish Central State. South European Society \& Politics, 12(3), 315-334, DOI: $10.1080 / 13608740701495186$

VALIENTE, CELIA (1994): El feminismo de Estado en España: El Instituto de la Mujer, 1983-1994. Working Paper 1994/58, Centro de Estudios Avanzados en Ciencias Sociales 
VERLOO, MIEKE ed. (2007): Multiple Meanings of Gender Equality. A Critical Frame Analysis of Gender Policies in Europe. Budapest: CEU Press

' Nota metodológica sobre la variable Color del partido político en el gobierno. Para ubicar ideológicamente en el eje derecha-izquierda a los partidos gobernantes a nivel central y autonómico se utilizaron los siguientes estudios postelectorales del Centro de Investigaciones Sociológicas (CIS): $\quad \quad N^{\circ} 2920$, Postelectoral Elecciones Generales, 2011; N 2.900 Postelectoral elecciones autonómicas y municipales, 2011 Comunidad de Cantabria; $\mathrm{N}^{\mathrm{o}} 2.896$, Postelectoral elecciones autonómicas y municipales, 2011, Comunidad Foral de Navarra; No 2709 Postelectoral elecciones Autonómicas 2007, Comunidad Autónoma de las Isles Baleares; №2964 Postelectoral elecciones autonómicas 2012, Comunidad Autónoma del País Vasco. En dichos estudios se incluye una pregunta en la cual se pide a la población entrevistada que ubique ideológicamente a los partidos políticos en una escala de 0 a 10, donde 0 corresponde al extremo de las posiciones de izquierda y 10 al extremo de la posiciones de derecha. En el caso de las coaliciones de gobierno se ha procedido a realizar la media ponderada de las puntuaciones obtenidas por cada partido según los estudios del CIS. Por ejemplo, si una coalición estaba formada por tres partidos, en primer lugar, en función del número de escaños que hubiesen obtenido se les asignó las posiciones de $1^{\circ}$ Partido, $2^{\circ}$ Partido y $3^{\circ}$ Partido dentro de la coalición de gobierno y, en segundo lugar, se les otorgó la puntuación que les correspondía a cada uno según los estudios del CIS y se aplicó la siguiente fórmula: (Puntuación $1^{\circ}$ Partido x 3)+ (Puntuación $2^{\circ}$ Partido x 2) $+\left(\right.$ Puntuación $3^{\circ}$ Partido x 1) / 6. Finalmente, una vez obtenidas todas las puntuaciones se categorizaron en una nueva escala de 1 a 5 , donde 1 equivale a la Derecha (puntuaciones 1 y 2 de los estudios del CIS), 2 al Centro-Derecha (puntuaciones 3 y 4 del CIS), 3 al Centro (puntuaciones 5 y (puntuaciones 7 y 8 del CIS) y 5 a la Izquierda (puntuaciones 9 Y 10 del CIS). 
En la siguiente tabla se muestran los partidos políticos y coaliciones de gobierno durante el periodo analizado, así como las puntuaciones obtenidas según los estudios del CIS y la categorización posterior que se realiza.

Tabla 5: Partidos en el gobierno durante el periodo analizado y ubicación ideológica de cada uno

\begin{tabular}{|c|c|c|c|c|}
\hline Partidos políticos en el gobierno & Estudio del CIS utilizado & $\begin{array}{l}\text { Puntuaciones } \\
\text { obtenidas en la } \\
\text { escala } 1-10\end{array}$ & $\begin{array}{l}\text { Categori } \\
\text { ideológic }\end{array}$ & ubicación \\
\hline Partido Popular (PP) & \multirow{5}{*}{$\begin{array}{l}\text { Estudio no } 2.920 \text { Postelectoral } \\
\text { elecciones generales } 2011\end{array}$} & 7,9 & 4 & $\begin{array}{l}\text { Centro- } \\
\text { Derecha }\end{array}$ \\
\hline $\begin{array}{l}\text { Partido Socialista Obrero Español } \\
\text { (PSOE) }\end{array}$ & & 4,1 & 2 & $\begin{array}{l}\text { Centro- } \\
\text { Izquierda }\end{array}$ \\
\hline Convergencia i Unió (CIU) & & 6,5 & 3 & Centro \\
\hline Coalición Canaria (CC) & & 5,9 & 3 & Centro \\
\hline $\begin{array}{l}\text { Partido Nacionalista Vasco (EAJ- } \\
\text { PNV) }\end{array}$ & & 6,2 & 3 & Centro \\
\hline Unión del Pueblo Navarro (UPN) & $\begin{array}{l}\text { Estudio no } 2.896 \text { Postelectoral } \\
\text { elecciones autonómicas y } \\
\text { municipales, } 2011 \text {. Comunidad } \\
\text { Foral de Navarra }\end{array}$ & 7,4 & 4 & $\begin{array}{l}\text { Centro- } \\
\text { Derecha }\end{array}$ \\
\hline
\end{tabular}


Tabla 6: Partidos en el gobierno durante el periodo analizado y ubicación ideológica

\begin{tabular}{|c|c|c|c|c|}
\hline $\begin{array}{l}\text { Coaliciones de partidos políticos } \\
\text { en el gobierno }\end{array}$ & Estudio del CIS utilizado & $\begin{array}{l}\text { Media } \\
\text { ponderada }\end{array}$ & $\begin{array}{l}\text { Categoriz } \\
\text { ideológic }\end{array}$ & Jbicación \\
\hline $\begin{array}{l}\text { Partido Socialista Obrero Español } \\
\text { (PSOE) - Bloc per Mallorca (BLOC) }\end{array}$ & $\begin{array}{l}\text { Estudio n } 2.920 \text { Postelectoral elecciones } \\
\text { generales } 2011 \text { / Estudio no } 2709 \\
\text { Postelectoral elecciones autonómicas } \\
\text { 2007. Comunidad Autónoma de las Islas } \\
\text { Baleares }\end{array}$ & 3,6 & 2 & Centro-Izquierda \\
\hline $\begin{array}{l}\text { Partido Socialista Obrero Español } \\
\text { (PSOE) - Bloc per Mallorca (BLOC) } \\
\text {-Unió Mallorquina (UM) }\end{array}$ & $\begin{array}{l}\text { Estudio no } 2.920 \text { Postelectoral elecciones } \\
\text { generales } 2011 \text { / Estudio no } 2709 \\
\text { Postelectoral elecciones autonómicas } \\
\text { 2007. Comunidad Autónoma de las Islas } \\
\text { Baleares }\end{array}$ & 4,0 & 2 & Centro-Izquierda \\
\hline $\begin{array}{l}\text { Partido Nacionalista Vasco (EAJ- } \\
\text { PNV) / Izquierda Unida-Ezker } \\
\text { Batua (EB) }\end{array}$ & $\begin{array}{l}\text { Estudio no } 2.920 \text { Postelectoral elecciones } \\
\text { generales } 2011 \text { /Estudio no } 2.964 \\
\text { Postelectoral elecciones autonómicas del } \\
\text { País Vasco } 2012 .\end{array}$ & 5,0 & 3 & Centro \\
\hline $\begin{array}{l}\text { Partido Popular (PP)-Partido } \\
\text { Regionalista de Cantabria (PRC) }\end{array}$ & $\begin{array}{l}\text { Estudio no } 2.920 \text { Postelectoral elecciones } \\
\text { generales 2011/ Estudio no } 2.900 \\
\text { Postelectoral elecciones autonómicas y } \\
\text { municipales, } 2011 \text {, Comunidad } \\
\text { autónoma de Cantabria }\end{array}$ & 7,0 & 4 & Centro-Derecha \\
\hline $\begin{array}{l}\text { Partido Socialista de Cataluña } \\
\text { (PSC)- Iniciativa Per Catalunya } \\
\text { (ICV)- Esquerra Republicana de } \\
\text { Catalunya (ERC) }\end{array}$ & $\begin{array}{l}\text { Estudio n } 2.920 \text { Postelectoral elecciones } \\
\text { generales } 2011\end{array}$ & 3,5 & 2 & Centro-Izquierda \\
\hline $\begin{array}{l}\text { PSdG (Partido Socialista de } \\
\text { Galicia) - Bloque Nacionalista } \\
\text { Galego (BNG) }\end{array}$ & $\begin{array}{l}\text { Estudio no } 2.920 \text { Postelectoral elecciones } \\
\text { generales } 2011\end{array}$ & 3,6 & 2 & Centro-Izquierda \\
\hline
\end{tabular}

